$\mathrm{E}$

EVALUAR
2020, Vol. 20, No. 2

ISSN $1667-4545$

Recuperado de https://revistas.unc.edu.ar/index.php/revaluar

Laboratorio de Evaluación Psicológica y Educativa

Facultad de Psicología - Universidad Nacional de Córdoba

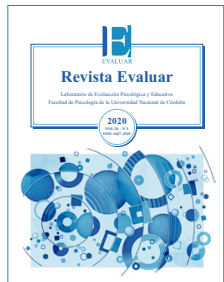

\title{
Escala de Adaptación Psicológica al Asesoramiento Genético (EAP-AG): validación de una versión en español para padres
}

\section{Psychological Adaptation Scale to Genetic Counseling: Validation of a Spanish Version for Parents}

Natalia Vázquez ${ }^{1,2}$ *, Javiera Ortega ${ }^{1,3}$, Kevin Scavone ${ }^{1}$, Virginia Corina Samaniego ${ }^{1}$, Claudia Arberas ${ }^{4}$
Introducción

Método

Resultados

Discusión

Referencias

1 - Centro de Investigaciones en Psicología y Psicopedagogía, Facultad de Psicología y Psicopedagogía, Pontificia Universidad Católica Argentina.

2 - Psicología aplicada a personas con enfermedades poco frecuentes (Paepof).

3 - Consejo Nacional de Investigaciones Cientificas y Técnicas CONICET.

4 - Sección de Genética Médica, Hospital de niños "Dr. Ricardo Gutiérrez".

Recibido: 08/04/2020 Revisado: 02/05/2020 Aceptado: 17/06/2020

\section{Resumen}

El objetivo de este trabajo es presentar la validación de un instrumento para medir en los padres la adaptación psicológica a una enfermedad o riesgo genético en sus hijos: Escala de Adaptación Psicológica al Asesoramiento Genético (EAP-AG). Se realizó un estudio metodológico, con una muestra de 203 casos. La validez de constructo fue estudiada mediante un análisis paralelo y un análisis factorial exploratorio. La significación del test de esfericidad de Bartlett $\left(\chi_{(105)}^{2}=1928.4 ; p<.001\right)$ fue significativa y el índice de adecuación muestral Kaiser-Meyer-Olkin (KMO) indicó una adecuación de .87. Se obtuvo un único factor que explicó el $45 \%$ de la varianza. La confiabilidad fue estudiada mediante el coeficiente alfa de Cronbach y se encontró un valor alto de consistencia interna $\alpha=.915$. El presente estudio ofrece el primer instrumento en español válido y confiable para medir en los padres la adaptación psicológica a la enfermedad o riesgo genético en sus hijos.

Palabras clave: validación, propiedades psicométricas, adaptación psicológica, asesoramiento genético

\section{Summary}

The objective of this work is to present the validation of an instrument to measure the psychological adaptation in parents to a disease or genetic risk in their children: Psychological Adaptation Scale to Genetic Counseling. A methodological study was carried out, with a sample of 203 cases. Construct validity was studied by a parallel analysis and an exploratory factor analysis. The significance of the Bartlett sphericity test $\left(\chi_{(105)}^{2}=1928.4 ; p<.001\right)$ was significant and the Kaiser-Meyer-Olkin (KMO) sample adequacy index indicated an adequacy of .87 . A single factor was obtained that explained $45 \%$ of the variance. Reliability was studied using the Cronbach's alpha coefficient, finding a high value of internal consistency $\alpha=.915$. The present study offers the first valid and reliable instrument in Spanish to measure, in parents, their psychological adaptation to a disease or genetic risk in their children.

Keywords: validation, psychometric properties, psychological adaptation, genetic counselling

*Correspondencia a: Natalia Vázquez. Av. Alicia Moreau de Justo 1300, C1107AAZ, Buenos Aires, Argentina 4349-0200 (int. 1107).

E-mail: natalia_vazquez@uca.edu.ar

Nota de autor: Esta investigación fue realizada en el marco de una Beca Postdoctoral de CONICET otorgada a la Dra. Natalia Vázquez. Agradecimiento: Se agradece a los Profesionales de la Sección de Genética Médica del Hospital de Niños Dr. Ricardo Gutiérrez, que brindaron su espacio y tiempo para el desarrollo de la investigación. A todos los padres y madres que generosa y desinteresadamente han participado del estudio.

Cómo citar este artículo: Vázquez, N., Ortega, J., Scavone, K., Samaniego, V. C., \& Arberas, C. (2020). Escala de Adaptación Psicológica al Asesoramiento Genético (EAP-AG): validación de una versión en español para padres. Revista Evaluar, 20(2), 20-34. 


\section{Introducción}

Es innegable que los niños diagnosticados con una enfermedad genética, y sus familias, merecen tener el apoyo psicológico necesario para que se puedan adaptar de la mejor manera a la situación. Sin embargo en Argentina, como en otras regiones de Latinoamérica, son escasos los estudios que miden el impacto psicológico que ocasionan las enfermedades genéticas a nivel familiar, y que diseñan programas o estrategias de intervención para contenerlo. Esto se debe, en parte, a la falta de instrumentos validados en español para medir la adaptación psicológica al diagnóstico o riesgo genético.

Las enfermedades genéticas varían en cuanto a su presentación clínica, morbilidad, mortalidad, prevalencia y oportunidades terapéuticas. Sin embargo, todas pueden generar un impacto psicológico a nivel familiar debido a las posibles dificultades en el logro del diagnóstico, a los constantes controles médicos, a los tratamientos exigentes y demandantes, y a la incertidumbre sobre la evolución de la enfermedad que se presentan en muchas ocasiones.

Desde la presunción hasta que el individuo recibe un diagnóstico específico de enfermedad genética, transcurre un proceso que pone en alerta tanto la salud del probando como la de su familia y entorno cercano. Si bien algunas familias logran manejar el estrés que provoca el proceso de recibir este diagnóstico, otras no logran adaptarse tan fácilmente. Por lo tanto, este factor se convierte en un estresor grave que puede provocar ansiedad, depresión y desesperanza ante la pérdida del control de la situación (Biesecker, 2010; Biesecker \& Peters, 2001; Dinc \& Terzioglu, 2006; Kessler, Kessler, Ward, \& Opitz, 1984; McAllister et al., 2008; Middleton, Hall, \& Patch, 2015; Middleton et al., 2014; Shiloh et al., 2014).

En esta línea, distintos autores introdujeron el término adaptación para referirse al proceso, que puede ser o no saludable, que la persona lleva a cabo, para hacer frente y ajustarse a vivir con esa enfermedad (Casier et al., 2013; Eiser, 1990; Shiloh, 1996).

Dado el marco del presente trabajo se utilizará la conceptualización realizada por Biesecker y Erby (2008) y Biesecker et al. (2013), quienes definen a la adaptación como un proceso y a su vez, un resultado. Es decir, se da con el tiempo y varía según los distintos momentos de la vida, el tiempo transcurrido desde el diagnóstico, el apoyo familiar y social, y las posibilidades de acceder a un proceso terapéutico. La adaptación psicológica es un constructo dinámico y multidimensional, capaz de abarcar tanto los aspectos emocionales como cognitivos del afrontamiento (Austin, Semaka, \& Hadjipavlou, 2014; Biesecker et al., 2013).

La escala Psychological Adaptation Scale (PAS; Biesecker et al., 2013) fue diseñada para evaluar en qué medida una familia se encuentra adaptada psicológicamente a una enfermedad genética o al riesgo genético de desarrollarla o transmitirla (Biesecker \& Erby, 2008). Los autores de la PAS plantean que la escala tiene cuatro dimensiones: afrontamiento eficaz, autoestima, integración social y bienestar espiritual/existencial. No realizan un desarrollo exhaustivo de cada una, pero, explicitan que la selección de los ítems para los dominios de afrontamiento, integración social y bienestar espiritual/existencial se obtuvieron del banco de ítems Patient-Reported Outcomes Measurement Information System (PROMIS) para medir el impacto psicológico de una enfermedad. Los ítems del dominio autoestima fueron adaptados de la Escala de Autoestima de Rosenberg (Austin et al., 2014; Biesecker \& Erby, 2008; Biesecker et al., 2013). También se refieren al modelo transaccional de afrontamiento del estrés de Lazarus y Folkman y a la teoría sobre 
la adaptación cognitiva de Taylor (Biesecker et al., 2013). La dimensión de afrontamiento es considerada un mediador de la adaptación en ambos modelos (Lazarus \& Folkman, 1984; Taylor, 1983), mientras que la recuperación de la autoestima, la búsqueda de sentido que lleva al bienestar existencial, y la reincorporación en la vida social se destacan en la teoría de Taylor por el rol fundamental que cumplen en la adaptación (Taylor, 1983). Sin embargo, debe señalarse como limitación que las cuatro dimensiones sugeridas por Biesecker et al. (2013) surgen de un análisis factorial confirmatorio (AFC), que no fue precedido por un análisis factorial exploratorio (AFE) de los ítems del instrumento. Los estudios que ponen a prueba la dimensionalidad de los instrumentos utilizan de manera privilegiada el procedimiento de análisis paralelo (AP) y AFE para determinar el número de factores (Auné \& Attorresi, 2017; De la Iglesia, Ongarato, \& Fernández-Liporace, 2010; Dominguez-Lara, \& Navarro-Loli, 2018).

Se ha realizado una búsqueda de antecedentes donde fue empleada la PAS (Biesecker et al., 2013) y surgen algunas consideraciones importantes (Berrios, Chakravarti, \& Biesecker, 2019; Edwards et al., 2018; Griesemer et al., 2019; Kortlever et al., 2019; Lamb et al., 2016; Park et al., 2016; Peay, Rosenstein, \& Biesecker, 2013; Peay, Rosenstein, \& Biesecker, 2014; Peay et al., 2016; Shapira et al., 2018; Truitt, Biesecker, Capone, Bailey, \& Erby, 2012; Turiff, Levy, \& Biesecker, 2015; Yanes, Humphreys, McInerneyLeo, \& Biesecker, 2016; Yopp et al., 2019). Hasta el momento, no se han encontrado estudios en que se haya utilizado la PAS (Biesecker et al., 2013) y se haya realizado un estudio acabado de sus propiedades psicométricas. En ninguno de los casos verificados se ha evaluado algún otro método de validez del instrumento, a excepción del estudio original, y en pocos casos se ha analizado su confiabilidad (Ver Tabla 1). Otro aspecto que llama la atención es el uso de la versión de la PAS de 20 ítems, pese a que Biesecker propone claramente la eliminación de 5 ítems luego de su AFC (Biesecker et al., 2013). Con respecto a los ámbitos de aplicación de la escala, esta fue originalmente creada para evaluar la adaptación psicológica dentro de un marco de asesoramiento genético para padres de niños con una condición genética como síndrome de Klinefelter, síndrome de Rett, distrofia muscular de Duchenne o trastorno del espectro autista. Sin embargo, la PAS (Biesecker et al., 2013) también ha sido utilizada con poblaciones no relativas al ámbito genético, destacando su uso con padres viudos y con personas con trastornos del estado del ánimo (considerando que atraviesan un proceso de adaptación psicológica). No obstante, su uso en estos nuevos ámbitos no ha venido acompañado de procedimientos de validación adecuados (ver Tabla 1).

Más allá de las limitaciones advertidas a nivel psicométrico, contar con un instrumento como este para medir la adaptación psicológica en la Argentina sería de gran valor. En términos de la investigación y el desarrollo de intervenciones psico-educativas, disponer de un instrumento en español para ser aplicado en ámbitos de asesoramiento genético sería de gran utilidad. Por ello, se decidió validar una versión en español para padres de la PAS (Biesecker et al., 2013), la Escala de Adaptación Psicológica al Asesoramiento Genético (EAP-AG).

Analizando la conceptualización de Biesecker et al. (2013), una buena adaptación psicológica podría funcionar como factor protector en la familia frente al diagnóstico o riesgo genético, indicando mayor resiliencia. Esclarecer la multi o unidimensionalidad del instrumento es relevante porque permitiría escoger el modo de abordaje psicológico para favorecer la adaptación.

El objetivo de este trabajo es presentar el proceso de validación de la EAP-AG como 
Tabla 1

Revisión de artículos que utilizaron la PAS.

\begin{tabular}{|c|c|c|c|c|c|}
\hline Cita & País & Población & $\begin{array}{l}\text { Versión } \\
\text { PAS }\end{array}$ & $\begin{array}{l}\text { Propiedades } \\
\text { psicométricas }\end{array}$ & Puntaje PAS \\
\hline $\begin{array}{l}\text { Park et al., } \\
2016\end{array}$ & $\begin{array}{l}\text { Estados } \\
\text { Unidos }\end{array}$ & $\begin{array}{l}308 \text { padres viudos con un hijo biológico o } \\
\text { adoptado menor a } 19 \text { años (dependent age } \\
\text { children) viviendo en su casa. La esposa (y } \\
\text { madre del hijo) murió por cáncer. }\end{array}$ & 15 ítems & No informa & $\mathrm{M}=3.2$ \\
\hline $\begin{array}{l}\text { Kortlever } \\
\text { et al., } 2019\end{array}$ & $\begin{array}{l}\text { Estados } \\
\text { Unidos }\end{array}$ & $\begin{array}{l}106 \text { adultos (pacientes) entre } 18 \text { y } 89 \text { años } \\
\text { con algún problema o enfermedad de } \\
\text { extremidades superiores (upper extremity } \\
\text { injury). }\end{array}$ & 20 ítem & No informa & $\mathrm{M}=49$ \\
\hline $\begin{array}{l}\text { Shapira et } \\
\text { al., } 2018\end{array}$ & $\begin{array}{l}\text { Estados } \\
\text { Unidos }\end{array}$ & $\begin{array}{l}103 \text { mujeres con una mutación en el gen } \\
\text { BRCA1/2 (gen de cáncer de mama/ovario) } \\
\text { y } 39 \text { parejas. }\end{array}$ & 15 ítems & $\begin{array}{l}\text { Solo informa alfa } \\
\text { de Cronbach }(.97 \\
\text { portadores, } .96 \\
\text { parejas) }\end{array}$ & $\begin{array}{c}\mathrm{M}=3.04 \text { para las } \\
\text { mujeres portadoras } \\
\text { y } \mathrm{M}=2.81 \text { para sus } \\
39 \text { parejas }\end{array}$ \\
\hline $\begin{array}{l}\text { Berrios et } \\
\text { al., } 2019\end{array}$ & $\begin{array}{l}\text { Estados } \\
\text { unidos }\end{array}$ & $\begin{array}{l}309 \text { adultos,padres de hijos con enfermedad } \\
\text { de Hirschprung. }\end{array}$ & 20 ítems & $\begin{array}{c}\text { Cita a Biesecker et } \\
\text { al., } 2013\end{array}$ & No informa \\
\hline $\begin{array}{l}\text { Truitt et } \\
\text { al., } 2012\end{array}$ & $\begin{array}{l}\text { Estados } \\
\text { Unidos }\end{array}$ & $\begin{array}{l}546 \text { padres (de } 21 \text { a } 84 \text { años) de hijos con } \\
\text { Síndrome de Down. }\end{array}$ & 20 ítems & $\begin{array}{l}\text { Alfa de Cronbach } \\
\text { (.94) }\end{array}$ & No informa \\
\hline $\begin{array}{l}\text { Peay et al., } \\
2016\end{array}$ & $\begin{array}{l}\text { Estados } \\
\text { Unidos }\end{array}$ & $\begin{array}{l}205 \text { madres de hijos con distrofia muscular } \\
\text { de Duchenne o Becker. }\end{array}$ & 20 ítems & $\begin{array}{c}\text { Cita a Biesecker et } \\
\text { al., } 2013\end{array}$ & $\mathrm{M}=3.5$ \\
\hline $\begin{array}{l}\text { Peay et al., } \\
2014\end{array}$ & $\begin{array}{l}\text { Estados } \\
\text { Unidos }\end{array}$ & $\begin{array}{l}266 \text { pacientes autoinformados como } \\
\text { bipolares (no se hizo evaluación } \mathrm{dx} \text { ). }\end{array}$ & 20 ítems & $\begin{array}{l}\text { Cita a Biesecker et } \\
\text { al., } 2013\end{array}$ & $M=2.6$ \\
\hline $\begin{array}{l}\text { Peay et al. } \\
2013\end{array}$ & $\begin{array}{l}\text { Estados } \\
\text { Unidos }\end{array}$ & 266 pacientes bipolares. & 20 ítems & $\begin{array}{c}\text { Cita a Biesecker et } \\
\text { al., } 2013\end{array}$ & $M=2.6$ \\
\hline $\begin{array}{l}\text { Griesemer } \\
\text { et al., } 2019\end{array}$ & $\begin{array}{l}\text { Estados } \\
\text { Unidos }\end{array}$ & $\begin{array}{l}192 \text { Pacientes con sospecha de diagnóstico } \\
\text { genético. }\end{array}$ & 15 ítems & $\begin{array}{c}\text { Cita a Biesecker et } \\
\text { al., } 2013\end{array}$ & No informa \\
\hline $\begin{array}{l}\text { Edwards et } \\
\text { al., } 2018\end{array}$ & $\begin{array}{l}\text { Estados } \\
\text { Unidos }\end{array}$ & $\begin{array}{l}204 \text { padres viudos con un hijo menor de } \\
18 \text { años. La madre(esposa del padre viudo) } \\
\text { murió de cáncer en los últimos } 5 \text { años. }\end{array}$ & $\begin{array}{l}\text { No in- } \\
\text { forma }\end{array}$ & No informa & No informa \\
\hline $\begin{array}{l}\text { Turriff et } \\
\text { al., } 2015\end{array}$ & $\begin{array}{l}\text { Estados } \\
\text { Unidos }\end{array}$ & $\begin{array}{l}250 \text { personas de } 14 \text { a } 75 \text { años con síndrome } \\
\text { de Klinefelter. }\end{array}$ & 20 ítems & $\begin{array}{c}\text { Cita a Biesecker et } \\
\text { al., } 2013\end{array}$ & $\mathrm{M}=3.33$ \\
\hline $\begin{array}{l}\text { Lamb et } \\
\text { al., } 2016\end{array}$ & $\begin{array}{l}\text { Estados } \\
\text { Unidos }\end{array}$ & $\begin{array}{l}388 \text { cuidadores primarios de hijos con } \\
\text { síndrome de Rett. }\end{array}$ & 20 ítems & $\begin{array}{c}\text { Cita a Biesecker et } \\
\text { al., } 2013\end{array}$ & $\mathrm{M}=3.80$ \\
\hline $\begin{array}{l}\text { Yopp et al., } \\
2019\end{array}$ & $\begin{array}{l}\text { Estados } \\
\text { Unidos }\end{array}$ & $\begin{array}{l}252 \text { personas viudas que perdieron a su } \\
\text { esposa en los últimos } 2 \text { años. }\end{array}$ & 15 ítems & $\begin{array}{c}\text { Cita a Biesecker et } \\
\text { al., } 2013\end{array}$ & No informa \\
\hline $\begin{array}{l}\text { Yanes et } \\
\text { al., } 2016\end{array}$ & $\begin{array}{l}\text { Australia, } \\
\text { Nueva } \\
\text { Zelanda } \\
\text { y Reino } \\
\text { unido }\end{array}$ & $\begin{array}{l}62 \text { padres de hijos con alguna condición } \\
\text { genética todavía sin diagnosticar }\end{array}$ & 20 ítems & $\begin{array}{c}\text { Cita a Biesecker et } \\
\text { al., } 2013\end{array}$ & $\mathrm{M}=2.89$ \\
\hline
\end{tabular}


instrumento para medir, en los padres, la adaptación psicológica a una enfermedad o riesgo genético en sus hijos. Presentamos el estudio de validez de constructo mediante un AP y un AFE, y el nivel de consistencia interna mediante el alfa de Cronbach.

\section{Método}

En el presente estudio metodológico se ha realizado la validación de la Escala de Adaptación Psicológica al Asesoramiento Genético (EAP$\mathrm{AG})$ para conocer en qué medida una familia se encuentra adaptada psicológicamente a una enfermedad genética, o al riesgo genético de desarrollarla o transmitirla.

En esta primera instancia, entonces, con la finalidad de aportar evidencia científica sobre las propiedades psicométricas del primer instrumento en español para medir la adaptación psicológica de los padres, se privilegió como medida de validez de constructo al AFE y como medida de confiabilidad al coeficiente alfa de Cronbach.

\section{Participantes}

La muestra final se compuso de 203 casos de madres y padres (mayormente madres 90.9\%) que informaron sus niveles de adaptación psicológica con respecto a la condición o riesgo genético de sus hijos (60.6\% varones y $39.4 \%$ mujeres, con un promedio de edad de $8.77, \mathrm{DE}=5.76)$. Se estudiaron familias con condiciones genéticas frecuentes como aquellas de niños con Enfermedades Neuromusculares (17.7\%), Síndrome de Down (13.3\%), Síndrome de Prader Willi (11.3\%), Retraso Global del Desarrollo (9.9\%), Trastorno del Espectro Autista (6.9\%), y en menor porcentaje, Mucopolisacaridosis $(2.5 \%)$, Cardiopatías congénitas (1.5\%), Neurofibromatosis tipo-I (2.5\%), Síndrome de Noonan (1.5\%), Síndrome de Charcot-Marie-Tooth (1.5\%), Síndrome de Moebius (1\%). E1 30.4\% restante fueron diagnósticos menos frecuentes en la consulta de genética durante el período en el que se relevaron los casos (baja talla, dismorfias y malformaciones aisladas, cromosomopatías, Síndrome de Bardet Biedl, entre otros). En la Tabla 2 se podrán ver, de manera detallada, las características de padres e hijos que respondieron el instrumento.

\section{Instrumentos}

Para participar del estudio era un requisito excluyente completar un formulario de consentimiento informado donde se les explicaba el propósito del estudio, y se indicaba que su participación consistía en completar cuestionarios, de manera voluntaria y anónima (se le asignó un número a cada protocolo). También se los informaba sobre sus derechos de acceder a los resultados del estudio y descontinuar su participación sin perjuicio alguno. El proyecto fue aprobado por el Comité de Ética en Investigación y el Comité de Docencia e Investigación del Hospital de Niños Dr. Ricardo Gutiérrez.

Para la evaluación del nivel socioeconómico se incluyeron preguntas del instrumento de la Asociación Argentina de Marketing (AAM, 2010) que incluye las siguientes categorías: situación laboral de ambos padres, nivel educativo, estado civil y cantidad de hijos en la familia.

Como instrumento de origen para realizar la adaptación psicológica se utilizó la Psychological Adaptation Scale (Biesecker et al., 2013). La PAS es un instrumento que Biesecker y colaboradores desarrollaron basados en las teorías de afrontamiento al estrés de Lazarus y Folkman y la teoría de adaptación cognitiva de Taylor. Originalmente 
Tabla 2

Distribución según variables sociodemográficas.

\begin{tabular}{lcc}
\hline \multicolumn{1}{c}{$\begin{array}{c}\text { Variables } \\
\text { sociodemográficas }\end{array}$} & $\mathbf{N}=\mathbf{2 0 3}$ \\
\hline $\begin{array}{l}\text { Características del hijo } \\
\text { Sexo del hijo }\end{array}$ & $\%$ & $\mathrm{~N}$ \\
Varones & $60.6 \%$ & 123 \\
Mujeres & $39.4 \%$ & 80 \\
& & \\
Edad & $\mathrm{M}=8.77 \quad \mathrm{DE}=5.76$ \\
& Mín $=0$ Máx $=35$
\end{tabular}

Escolaridad

No concurre a ningún tipo

Jardín maternal o guardería

Jardín de infantes

$15.3 \%$

26

$4.7 \%$

$7.6 \%$

$7.6 \%$

$40.0 \%$

$5.9 \%$

$18.8 \%$

$\mathrm{M}=3.24 \mathrm{DE}=3.98$

Mín $=0$ Máx $=16$

\section{Características}

de los padres

Sexo del cuidador que

respondió el cuestionario

Masculino

$9.1 \%$

10

Femenino

Lugar de procedencia

Ciudad Autónoma de

Buenos Aires

$20.3 \%$

40

Provincia de Buenos Aires

Otra provincia

$64.5 \%$

127

$15.2 \%$

30

Edad de la madre

$\mathrm{M}=38.74 \mathrm{DE}=8.88$

Mín $=19$ Máx $=64$

Edad del padre

$\mathrm{M}=42.14 \mathrm{DE}=9.98$

Mín $=20$ Máx $=73$ el instrumento fue diseñado con 20 ítems, con opciones de respuesta tipo Likert, para medir cuatro dominios de la adaptación psicológica: afrontamiento eficaz, autoestima, integración social y bienestar espiritual/existencial. Biesecker y colaboradores a partir de un análisis factorial confirmatorio decidieron eliminar 5 ítems quedando así una versión de 15 ítems (Biesecker et al., 2013). A partir de una comunicación personal con la autora principal del instrumento se decidió validar en Argentina la versión reducida de 15 ítems.

La escala puede ser respondida tanto por la persona afectada por la condición o riesgo genético como por sus padres o cuidadores. En ambas situaciones, es importante tener en consideración que hayan pasado al menos 6 meses desde la identificación del diagnóstico o riesgo genético, porque la adaptación psicológica es un proceso que requiere de tiempo. Si responde la persona afectada, es importante considerar su edad: no se recomienda la aplicación de esta prueba en individuos que no hayan alcanzado la adolescencia, debido a que podrían presentarse dificultades en la comprensión de los ítems. En el caso de que fuera posible, contar con múltiples informantes (ambos padres o la dupla adolescente/adulto y padres) permitiría tener una comprensión más global del impacto a nivel familiar.

Las consignas del cuestionario piden al evaluado indicar si vivir con una condición genética o ser cuidador de un niño que la posee lo ha ayudado en diferentes aspectos. El participante debe señalar para cada consigna el grado de acuerdo: (1) de ningún modo, (2) un poco, (3) algo, (4) bastante, (5) mucho. Con respecto a la interpretación de los valores que pueden obtenerse a través de este instrumento, cuanto mayor es el puntaje mejor es el nivel de adaptación psicológica. Los autores determinaron como puntaje de corte para establecer un buen nivel de adaptación psicológica el valor de 3. El puntaje final se obtiene dividiendo las 


\begin{tabular}{|c|c|c|}
\hline $\begin{array}{c}\text { Variables } \\
\text { sociodemográficas }\end{array}$ & \multicolumn{2}{|c|}{$\mathbf{N}=\mathbf{2 0 3}$} \\
\hline \multicolumn{3}{|l|}{ Estado civil de los padres } \\
\hline Casados o viviendo juntos & $73.4 \%$ & 130 \\
\hline Separados o divorciados & $17.0 \%$ & 30 \\
\hline Madre sola & $7.9 \%$ & 14 \\
\hline Viudo(a) & $1.7 \%$ & 3 \\
\hline \multicolumn{3}{|l|}{$\begin{array}{l}\text { Máx. nivel de instrucción: } \\
\text { madre }\end{array}$} \\
\hline Secundario incompleto & $38.2 \%$ & 73 \\
\hline Secundario completo & $28.8 \%$ & 51 \\
\hline $\begin{array}{l}\text { Terciario incompleto/ } \\
\text { completo }\end{array}$ & $13.0 \%$ & 23 \\
\hline $\begin{array}{l}\text { Universitario incompleto/ } \\
\text { completo }\end{array}$ & $16.4 \%$ & 29 \\
\hline Sin estudios & $0.60 \%$ & 1 \\
\hline \multicolumn{3}{|l|}{$\begin{array}{l}\text { Máx. nivel de instrucción: } \\
\text { padre }\end{array}$} \\
\hline Secundario incompleto & $47.00 \%$ & 87 \\
\hline Secundario completo & $27.60 \%$ & 56 \\
\hline $\begin{array}{l}\text { Terciario incompleto/ } \\
\text { completo }\end{array}$ & $33.50 \%$ & 20 \\
\hline $\begin{array}{l}\text { Universitario incompleto/ } \\
\text { completo }\end{array}$ & $10.30 \%$ & 29 \\
\hline
\end{tabular}

\section{Principal sostén económico} familiar

Persona con mayores

ingresos

Padre

$53.8 \%$

Madre

$32.4 \%$

$13.7 \%$

Laboralmente activo

$91.50 \%$

Cobertura médica

\begin{tabular}{lcc} 
Sí & $60.7 \%$ & 122 \\
No & $37.8 \%$ & 76 \\
No sabe-No contesta & $1.5 \%$ & 3 \\
\hline
\end{tabular}

puntuaciones obtenidas por la cantidad de ítems, tanto para cada dimensión como para el puntaje total. Los puntajes por dimensión permiten conocer en qué dominios las personas tienen un mejor o peor nivel de adaptación psicológica.

Como se mencionó anteriormente no existen, hasta el momento, versiones de la PAS (Biesecker et al., 2013) para población de habla hispana. Por lo tanto, se realizó una adaptación lingüística al español de la versión original en inglés con el nombre de Escala de Adaptación Psicológica al Asesoramiento Genético (EAP$A G)$. Se revisaron aspectos de equivalencia de contenido, equivalencia semántica y validez de contenido. Como estrategia de traducción se siguió la metodología que suele ser empleada para instrumentos que miden calidad de vida y que aventaja al modelo clásico de traducción inversa (World Health Organization, 2009). El instrumento fue traducido del inglés al español por dos traductores que se consultaron y trabajaron de manera colaborativa. Luego, un panel bilingüe de 4 personas capaces de hablar y escribir en inglés y español revisó la traducción buscando inconsistencias entre la versión original en inglés y el documento traducido en español. Por otro lado, un grupo monolingüe de 4 personas representativas de quienes usarían el instrumento, no familiarizados con el mismo, se encargaron de buscar expresiones del lenguaje que no resultasen claras o fueran ambiguas. También se realizó un grupo focal integrado por becarios y adscriptos del Centro de Investigaciones en Psicología y Psicopedagogía, moderado por un miembro del panel bilingüe. El grupo bilingüe consideró los comentarios del grupo monolingüe y se incorporaron en el documento traducido las modificaciones sugeridas por este último grupo, toda vez que tuvieron relación con el texto de la versión original. Luego el documento atravesó un proceso de traducción inversa al lenguaje original, realizada por un traductor que no 
conocía la versión original en inglés. Finalmente, el grupo bilingüe comparó la versión original del instrumento y la versión que resultó de la traducción inversa, buscando diferencias significativas en cuanto a equivalencia conceptual, semántica y técnica. Se hicieron estudios piloto en una muestra dentro del servicio de genética médica de la misma institución donde se realizaría la validación, a fin de ajustar la redacción de los ítems, corroborando que fueran comprendidos por la población destinataria.

\section{Procedimiento}

Se empleó un muestreo de tipo no probabilístico intencional. El $71 \%$ de los casos fue recabado de manera presencial por un psicólogo en la Sección de Genética Médica del Hospital de Niños Dr. Ricardo Gutiérrez, entre el año 2017 y el año 2019. Otra parte de la muestra (29\%) fue recabada en una modalidad virtual, con aplicación autoadministrada de los cuestionarios, a través de grupos online de apoyo de padres con niños, adolescentes, o adultos (en menor proporción), con diagnóstico de Enfermedades Poco Frecuentes (EPF).

\section{Tratamiento estadístico de los datos}

Para realizar los estudios de validez de la primera versión en español de la escala EAP-AG se decidió, en primer lugar, realizar la implementación óptima del AP (Timmerman \& LorenzoSeva, 2011), un procedimiento más actualizado que el propuesto por Horn (1965). Tal como sugieren Pérez-Gil, Chacón-Moscoso y MorenoRodríguez (2000), se evaluó la composición factorial sin tener en cuenta la estructura de 4 factores que habían informado los autores originales de la escala. De este modo se buscó determinar el número de dimensiones. En segundo lugar, se decidió hacer un AFE. Para realizar ambos procedimientos se utilizó el programa FACTOR 9.2 (Lorenzo-Seva \& Ferrando, 2013).

Para los análisis descriptivos y de confiabilidad de la primera versión en español de la EAPAG se utilizó el SPSS versión 25 (IBM, 2017). La consistencia interna se analizó mediante el cálculo de los coeficientes alfa de Cronbach, considerando índices excelentes a los coeficientes superiores a .90 , buenos a los superiores a .80, y aceptables a los superiores a .70 (George \& Mallery, 2001).

\section{Resultados}

Estudios de Validez y Confiabilidad de la $E A P-A G$

La validez del instrumento fue estudiada mediante el método de análisis factorial. Siguiendo los criterios de Martínez-Arias (1995), la cantidad de observaciones realizadas es suficiente para realizar el AFE. Estimando necesarios al menos 5 casos por ítem, y habiendo administrado la versión de 15 ítems de la EAP-AG, se trabajó con una muestra de 203 casos.

Se realizó un AP para determinar el número de factores. Este análisis sugirió una estructura de un único factor, extraído por el método de mínimos cuadrados no ponderados (ULS), recomendado para estos casos en los que no se cuenta con una distribución normal de los datos (Forero, Maydeu-Olivares, \& Gallardo-Pujol, 2009).

Luego se procedió a realizar un AFE con el único factor que sugirió el análisis paralelo. Se utilizó la rotación promax para mejorar la interpretación de los resultados (Fabrigar, Wegener, MacCallum, \& Strahan, 1999). El resultado del test de esfericidad de Bartlett $\left(\chi_{(105)}^{2}=1928.4 ; p<.001\right)$ fue estadísticamente 
Tabla 3

Ítems de la Escala de Adaptación Psicológica al Asesoramiento Genético (EAP-AG).

\begin{tabular}{|c|c|c|c|c|c|c|}
\hline Variable & Media & DE & Asimetría & Curtosis & $\begin{array}{c}\text { Peso } \\
\text { factorial }\end{array}$ & $\begin{array}{c}\text { Alfa si se } \\
\text { elimina el } \\
\text { elemento }\end{array}$ \\
\hline $\begin{array}{l}\text { EAP-AG1 PAS1 Me ayudó a aceptar la forma en } \\
\text { que las cosas suceden. }\end{array}$ & 4.39 & 1.05 & -1.86 & 2.82 & .546 & .912 \\
\hline $\begin{array}{l}\text { EAP-AG2 PAS2 Me ayudó a aprender a lidiar } \\
\text { mejor con la incertidumbre. }\end{array}$ & 4.24 & 1.18 & -1.49 & 1.17 & .462 & .914 \\
\hline $\begin{array}{l}\text { EAP-AG3 PAS3 Me enseñó a adaptarme a las } \\
\text { cosas que no puedo cambiar. }\end{array}$ & 4.31 & 1.11 & -1.58 & 1.59 & .487 & .914 \\
\hline $\begin{array}{l}\text { EAP-AG4 PAS4 Me ayudó a tomar las cosas tal } \\
\text { como vienen. }\end{array}$ & 4.46 & .99 & -1.90 & 2.85 & .524 & .912 \\
\hline $\begin{array}{l}\text { EAP-AG5 PAS6 Me ayudó a aprender a manejar } \\
\text { tiempos difíciles. }\end{array}$ & 4.40 & 1.02 & -1.63 & 1.59 & .595 & .911 \\
\hline $\begin{array}{l}\text { EAP-AG6 PAS7 Me ayudó a sentirme más } \\
\text { cómodo con quien soy. }\end{array}$ & 4.19 & 1.24 & -1.45 & .99 & .715 & .906 \\
\hline $\begin{array}{l}\text { EAP-AG7 PAS8 Me ayudó a ser una persona } \\
\text { más fuerte. }\end{array}$ & 4.61 & .92 & -2.52 & 5.68 & .696 & .907 \\
\hline $\begin{array}{l}\text { EAP-AG8 PAS9 Me ayudó a sentirme mejor } \\
\text { con mi capacidad para manejar los problemas. }\end{array}$ & 4.49 & .94 & -1.93 & 2.97 & .586 & .910 \\
\hline $\begin{array}{l}\text { EAP-AG9 PAS10 Me ayudó a ser una mejor } \\
\text { persona. }\end{array}$ & 4.28 & 1.20 & -1.69 & 1.76 & .620 & .911 \\
\hline $\begin{array}{l}\text { EAP-AG10 PAS13 Ayudó a que las relaciones } \\
\text { sean más significativas. }\end{array}$ & 4.42 & 1.10 & -1.92 & 2.72 & .699 & .908 \\
\hline $\begin{array}{l}\text { EAP-AG11 PAS14 Me ayudó a estar más cerca } \\
\text { de las personas que me importan. }\end{array}$ & 4.50 & 1.07 & -2.31 & 4.42 & .671 & .910 \\
\hline $\begin{array}{l}\text { EAP-AG12 PAS15 Me ayudó a ser más } \\
\text { consciente del amor y el apoyo disponible de } \\
\text { otras personas. }\end{array}$ & 4.56 & 1.01 & -2.50 & 5.41 & .592 & .912 \\
\hline $\begin{array}{l}\text { EAP-AG13 PAS16 Me ayudó a aprender que mi } \\
\text { vida es significativa. }\end{array}$ & 4.52 & 1.00 & -2.25 & 4.37 & .801 & .906 \\
\hline $\begin{array}{l}\text { EAP-AG14 PAS17 Me ha dado un mayor } \\
\text { aprecio por la vida. }\end{array}$ & 4.56 & .96 & -2.38 & 5.11 & .790 & .906 \\
\hline $\begin{array}{l}\text { EAP-AG15 PAS18 Me ayudó a desarrollar un } \\
\text { sentido más profundo del propósito en la vida. }\end{array}$ & 4.52 & .99 & -2.30 & 4.74 & .763 & .907 \\
\hline
\end{tabular}

significativo y el índice de adecuación muestral Kaiser-Meyer-Olkin (KMO) indicó una adecuación de .87, datos que sugieren, respectivamente, una buena correlación entre los ítems y una buena adecuación muestral, y que evidencian la pertinencia de un análisis factorial
(Kaiser \& Raice, 1974). El factor obtenido permitió explicar el 45\% de la varianza.

Para estudiar la confiabilidad del instrumento se analizó su consistencia interna mediante el cálculo del coeficiente alfa de Cronbach. Para la versión en español de 15 ítems el valor obtenido 
fue de $\alpha=.915$, que según los parámetros considerados evidencia un excelente nivel de confiabilidad. Además, se verificó que en ningún caso mejora este coeficiente si se elimina algún elemento.

En la Tabla 3 puede consultarse información relevante de cada ítem de la escala: valores de media, desvío, asimetría, curtosis, peso factorial $\mathrm{y}$ valor de alfa si se elimina el elemento.

\section{Discusión}

La investigación realizada ofrece un estudio inicial de las propiedades psicométricas de la Escala de Adaptación Psicológica al Asesoramiento Genético (EAP-AG), diseñada para ser respondida por padres o cuidadores en población argentina.

Se trata de la primera adaptación lingüística que se realiza del instrumento. Los estudios anteriores que utilizaron la PAS (Biesecker et al., 2013) lo hicieron con la escala en su idioma original inglés, por tratarse de estudios realizados, mayormente, en Estados Unidos, o Australia y Nueva Zelanda (Berrios et al., 2019; Edwards et al., 2018; Griesener et al., 2019; Shapira et al., 2018; Lamb et al., 2016; Peay et al., 2013, 2014; 2016; Turriff, Levy, \& Biesecker, 2015; Truitt et al, 2012; Yanes et al., 2017).

El presente estudio ofreció por primera vez un análisis paralelo y un análisis factorial exploratorio de la escala. Los resultados se contraponen con lo planteado por Biesecker et al. (2013). $\mathrm{Si}$ bien Biesecker, a partir del AFC, informa cuatro dimensiones de la PAS (afrontamiento eficaz, autoestima, integración social y bienestar espiritual/existencial), los análisis de este estudio no han aportado evidencia para considerar que la escala sea multidimensional. Se obtuvo en cambio una estructura de un único factor capaz de explicar el $45 \%$ de la varianza, es decir que la EAP-AG se comportaría como unidimensional. Hasta el momento se puede mencionar a favor de los resultados aquí expuestos que el AFE es la técnica utilizada por excelencia para el desarrollo y validación de instrumentos (Auné, \& Attorresi, 2017; De la Iglesia et al., 2010; Dominguez-Lara, \& Navarro-Loli, 2018; LloretSegura, Ferreres-Traver, Hernández-Baeza, \& Tomás-Marco, 2014). Una posible interpretación de estas diferencias podría indicar que la adaptación psicológica es un fenómeno complejo, con varios aspectos que la componen (afrontamiento eficaz, autoestima, integración social y bienestar espiritual/existencial), pero sin ser dimensiones individuales, sino aspectos de una única dimensión. Esta situación también se evidenció al administrar la escala, momento en que se hizo aparente que aquellos padres que tenían un buen nivel de adaptación psicológica puntuaban de manera semejante (elevado) en todos los ítems. En contraposición, los padres que tenían un bajo nivel de adaptación psicológica puntuaban bajo en todos los ítems.

Con relación a su confiabilidad, la EAP-AG ha demostrado ser un instrumento con un excelente nivel de consistencia interna ( $\alpha=.915)$ para la población argentina, obteniendo valores superiores a los del instrumento original ( $>.80$; Biesecker et al., 2013) y a los reportados por Truitt et al. (2012) y Klein et al. (2018). El coeficiente alfa de Cronbach es considerado el método privilegiado para analizar la confiabilidad de un instrumento (Raykov \& Marcoulides, 2019).

El presente estudio tiene limitaciones importantes para mencionar. La fuerte contradicción entre los hallazgos, respecto de la unidimensionalidad versus la multidimensionalidad de la escala, sin lugar a duda exige nuevos análisis para comprender en mayor profundidad las propiedades psicométricas de esta escala. Debido a la cantidad de casos no ha sido posible contar con 
otra muestra para realizar un AFC posterior al AFE en esta instancia. Sin embargo, se buscará realizar este análisis considerando datos que actualmente se están relevando con esta versión en español. Por otro lado, si bien la confiabilidad se ha estudiado con el método más ampliamente utilizado en estudios psicométricos, el análisis de la consistencia interna, sería oportuno que futuros estudios pudieran evaluarla mediante análisis de test-retest y estabilidad a largo plazo. Otro aspecto importante es la falta de mediciones de efecto techo o efecto suelo en las puntuaciones. Serían oportunos análisis de sensibilidad y especificidad por curvas ROC en futuros estudios.

Uno de los puntos fuertes de la EAP-AG es que permite medir un constructo relevante para el asesoramiento genético, como lo es la adaptación psicológica, de una manera sencilla y rápida. $\mathrm{La}$ claridad de los ítems destaca como una ventaja de este instrumento: personas con primaria incompleta o sin estudios pudieron responder a toda la escala sin dificultad. Esto es importante para su aplicación en el ámbito de los hospitales públicos, donde suelen asistir las familias con niveles más bajos de educación. Además, la escala es adecuada para ser aplicada en consultas médicas o psicológicas, resulta económica, y de duración breve (15 minutos). Es una escala autoadministrable, adolescentes y adultos con riesgo o diagnóstico genético, familiares y cuidadores pueden responderla informando sus propios niveles de adaptación psicológica. Sin embargo, es aconsejable que el profesional sea quien administre la escala, porque esta instancia se convierte en una oportunidad única de encuentro con la familia para conversar sobre la experiencia subjetiva respecto a su diagnóstico o riesgo genético.

Como sus autores han planteado, uno de los principales usos de este instrumento es el de identificar la necesidad de una intervención psicológica cuando los niveles de adaptación son ba- jos (puntajes promedio menores a 3). También es recomendable, pasado un tiempo, ofrecerle a las familias la posibilidad de responder nuevamente la escala porque tal como se ha mencionado, la adaptación psicológica es un proceso que puede variar en el tiempo. De este modo se podrá reflexionar sobre los avances y cambios producidos en la adaptación psicológica al diagnóstico.

A modo de conclusiones generales, el presente estudio resulta un aporte metodológico altamente relevante. Se han aportado evidencias de confiabilidad y validez de la EAP-AG para su aplicación en Argentina. Se sugiere su uso para el desarrollo de investigaciones en el campo del asesoramiento genético. La disponibilidad de este instrumento permitirá, además, conocer el estado de adaptación psicológica de las familias de habla hispana con riesgo o diagnóstico genético.

En muchas oportunidades, la ausencia de instrumentos validados en español hace difícil la producción de conocimiento científico en nuestro contexto. Además, el campo del asesoramiento genético es un ámbito ignorado por muchos psicólogos. Por ello, este esfuerzo es significativo debido a las posibilidades que brindará la medición de la adaptación psicológica para la posterior intervención psicológica en este campo.

A modo de implicancias, la disponibilidad del instrumento permitirá reducir la brecha existente entre el impacto psicológico del diagnóstico y la derivación de la familia a un tratamiento psicológico oportuno, ya que posibilitará identificar las necesidades de intervención. También puede ser útil como una herramienta que mida la eficacia de programas de intervención o progreso terapéutico. 


\section{Referencias}

Asociación Argentina de Marketing (2010). Índice de nivel socio económico 1996. Asociación Argentina de Marketing, Comisión de Investigación de Mercado. Recuperado de https://www.taller-de-radio.com.ar/ files/Nivel_Socio_Economico.pdf

Auné, S. E., \& Attorresi, H. F. (2017). Dimensionalidad de un Test de Conducta Prosocial. Revista Evaluar, 17(1), 29-37. doi: 10.35670/1667-4545.v17. n1.17072

Austin, J., Semaka, A., \& Hadjipavlou, G. (2014). Conceptualizing genetic counseling as psychotherapy in the era of genomic medicine. Journal of Genetic Counseling, 23(6), 903-909. doi: 10.1007/ s10897-014-9728-1

Berrios, C. D., Chakravarti, A., \& Biesecker, B. B. (2019). High levels of interest in reproductive genetic information in parents of children and adults with Hirschsprung disease. Journal of Pediatric Gastroenterology and Nutrition, 69(3), 299-305. doi: 10.1097/MPG.0000000000002392

Biesecker, B. B. (2010). Genetic Counselling: Psychological Issues. En Encyclopedia of Life Sciences (ELS). Chichester, UK: John Wiley \& Sons. doi: 10.1002/9780470015902.a0005616.pub2

Biesecker, B. B., \& Erby, L. (2008). Adaptation to living with a genetic condition or risk: A mini review. Clinical Genetics, 74(5), 401-407. doi: 10.1111/j.1399-0004.2008.01088.x

Biesecker, B. B., \& Peters, K. F. (2001). Process studies in genetic counseling: Peering into the black box. American Journal of Medical Genetics, 106(3), 191198. doi: 10.1002/ajmg.10004

Biesecker, B. B., Erby, L. H., Woolford, S., Adcock, J. Y., Cohen, J. S., Lamb, A., ...\& Reeve, B. B. (2013). Development and validation of the Psychological Adaptation Scale (PAS): Use in six studies of adaptation to a health condition or risk. Patient Education and Counseling, 93(2), 248-254. doi: 10.1016/j. pec.2013.05.006
Casier, A., Goubert, L., Gebhardt, W. A., De Baets, F., Van Aken, S., Matthys, D., \& Crombez, G. (2013). Acceptance, well-being and goals in adolescents with chronic illness: a daily process analysis. Psychology \& Health, 28(11), 1337-1351. doi: 10.1080/08870446.2013.809083

De la Iglesia, G., Ongarato, P., \& Fernández-Liporace, M. (2010). Propiedades psicométricas de una Escala de Estilos Parentales e Inconsistencia Parental Percibida (EPIPP). Revista Evaluar, 10(1), 32-52. doi: 10.35670/1667-4545.v10.n1.455

Dinc, L., \& Terzioglu, F. (2006). The psychological impact of genetic testing on parents. Journal of Clinical Nursing, 15(1), 45-51. doi: 10.1111/j.1365-2702.2005.01228.x

Dominguez-Lara, S., \& Navarro-Loli, J. S. (2018). Revisión de metodologías empleadas en los estudios factoriales de la Escala de Bienestar Psicológico de Ryff (versión en español). Revista Evaluar, 18(2), 17-30. doi: 10.35670/1667-4545.v18.n2.20800

Edwards, T. P., Yopp, J. M., Park, E. M., Deal, A., Biesecker, B. B., \& Rosenstein, D. L. (2018). Widowed parenting self-efficacy scale: A new measure. Death Studies, 42(4), 247-253. doi: 10.1080/07481187.2017.1339743

Eiser, C. (1990). Chronic Childhood Disease. An Introduction to Psychological Theory and Research. Cambridge, UK: Cambridge University Press.

Fabrigar, L. R., Wegener, D. T., MacCallum, R. C., \& Strahan, E. J. (1999). Evaluating the use of exploratory factor analysis in psychological research. Psychological Methods, 4(3), 272-299. doi: 10.1037/1082-989X.4.3.272

Gallardo-Pujol, D., Forero, C. G., Maydeu-Olivares, A., \& Andrés-Pueyo, A. (2009). Desarrollo del comportamiento antisocial: Factores psicobiológicos, ambientales e interacciones genotipo-ambiente. Revista de Neurología, 48(4), 191-198.

George, D., \& Mallery, P. (2001). SPSS for windows step by step: A simple guide and reference. Boston: Allyn and Bacon. 
Griesemer, I., Moore, E., Khan, C., Roche, M., Henderson, G., \& Rini, C. (2019). Psychological adaptation to diagnostic genomic sequencing results: The role of hope fulfillment. Health Psychology, 38(6), 527-535. doi: $10.1037 /$ hea0000733

Horn, J. L. (1965). A rationale and test for the number of factors in factor analysis. Psychometrika, 30(2), 179-185.

IBM Corp. (2017). IBM SPSS Statistics for Windows, Version 25.0. [Software de cómputo]. Armonk, NY: IBM Corp.

Kaiser, H. F., \& Rice, J. (1974). Little Jiffy, Mark IV. Educational and Psychological Measurement, 34(1), 111-117. doi: 10.1177/001316447403400115

Kessler, S., Kessler, H., Ward, P., \& Opitz, J. M. (1984). Psychological aspects of genetic counseling, III. Management of guilt and shame. American Journal of Medical Genetics, 17(3), 673-697. doi: 10.1002/ ajmg. 1320170320

Kortlever, J. T. P., Keulen, M. H. F., Teunis, T., Ring, D., Driscoll, M. D., Reichel, L. M., \& Vagner, G. A. (2019). Does resiliency mediate the association of psychological adaptability with disability and pain in patients with an upper extremity injury or illness? Journal of Psychosomatic Research, 117, 1-9. doi: 10.1016/j.jpsychores.2018.12.002

Lamb, A. E., Biesecker, B. B., Umstead, K. L., Muratori, M., Biesecker, L. G., \& Erby, L. H. (2016). Family functioning mediates adaptation in caregivers of individuals with Rett syndrome. Patient Education and Counseling, 99(11), 1873-1879. doi: 10.1016/j. pec. 2016.06 .018

Lazarus, R. S., \& Folkman, S. (1984). Stress, appraisal, and coping. New York, NY: Springer.

Lloret-Segura, S., Ferreres-Traver, A., Hernández-Baeza, A., \& Tomás-Marco, I. (2014). El análisis factorial exploratorio de los ítems: Una guía práctica, revisada y actualizada. Anales de Psicología, 30(3), 11511169. doi: 10.6018/analesps.30.3.199361

Lorenzo-Seva, U., \& Ferrando, P. J. (2013). FACTOR 9.2: A comprehensive program for fitting exploratory and semiconfirmatory factor analysis and IRT models. Applied Psychological Measurement, 37(6), 497498. doi: 10.1177/0146621613487794

Martínez-Arias, R. (1995). El método de encuestas por muestreo: Conceptos básicos. En M. T. Anguera, J. Arnau, M. Ato, R. Martínez Arias, J. Pascual y G. Vallejo, Métodos de investigación en Psicología. Madrid: Síntesis.

McAllister, M., Payne, K., Macleod, R., Nicholls, S., Donna, D., \& Davies, L. (2008). Patient empowerment in clinical genetics services. Journal of Health Psychology, 13(7) 895-905. doi: 10.1177/1359105308095063

Middleton, A., Hall, G., \& Patch, C. (2015). Genetic counselors and genomic counseling in the United Kingdom. Molecular Genetics \& Genomic Medicine, 3(2), 79-83. doi: 10.1002/mgg3.123

Middleton, A., Patch, C., Wiggins, J., Barnes, K., Crawford, G., Benjamin, C., \& Bruce, A. (2014). Position statement on opportunistic genomic screening from the Association of Genetic Nurses and Counsellors (UK and Ireland). European Journal of Human Genetics, 22(8), 955-956. doi: 10.1038/ejhg.2013.301

Park, E. M., Deal, A. M., Yopp, J. M., Edwards, T. P., Wilson, D. J., Hanson, L. C., \& Rosenstein, D. L. (2016). End-of-life experiences of mothers with advanced cancer: Perspectives of widowed fathers. BMJ Supportive \& Palliative Care, 6(4), 437-444. doi: 10.1136/bmjspcare-2015-000976

Peay, H. L., Meiser, B., Kinnett, K., Furlong, P., Porter, K., \& Tibben, A. (2016). Mothers' psychological adaptation to Duchenne/Becker muscular dystrophy. European Journal of Human Genetics, 24(5), 633637. doi: 10.1038/ejhg.2015.189

Peay, H. L., Rosenstein, D. L. \& Biesecker, B. B. (2013). Adaptation to bipolar disorder and perceived risk to children: A survey of parents with bipolar disorder. BMC Psychiatry, 13(1), 327. doi: 10.1186 / $1471-244 x-13-327$

Peay, H. L., Rosenstein, D. L., \& Biesecker, B. B. (2014). Parenting with bipolar disorder: Coping with risk of mood disorders to children. Social 
Science \& Medicine, 104, 194-200. doi: 10.1016/j. socscimed.2013.10.022

Pérez-Gil, J. A. P., Chacón-Moscoso, S. C., \& MorenoRodríguez, R. M. (2000). Validez de constructo: El uso de análisis factorial exploratorio-confirmatorio para obtener evidencias de validez. Psicothema, 12(Sup.2), 442-446. Recuperado de https://www.psicothema.com

Raykov, T., \& Marcoulides, G. A. (2019). Thanks coefficient alpha, we still need you! Educational and Psychological Measurement, 79(1), 200-210. doi: 10.1177/0013164417725127

Shapira, R., Turbitt, E., Erby, L. H., Biesecker, B. B., Klein, W. M. P., \& Hooker, G. W. (2018). Adaptation of couples living with a high risk of breast/ovarian cancer and the association with risk-reducing surgery. Familial Cancer, 17(4), 485-493. doi: 10.1007/ s10689-017-0065-Z

Shiloh, S. (1996). Genetic counseling: A developing area of interest for psychologists. Professional Psychology: Research and Practice, 27(5), 475-486. doi: 10.1037/0735-7028.27.5.475

Shiloh, S., deHeer, H. D., Peleg, S., Hensley-Alford, S., Skapinsky, K., Roberts, J. S., \& Hadley, D. W. (2014). The impact of multiplex genetic testing on disease risk perceptions. Clinical Genetics, 87(2), 117-123. doi: 10.1111/cge.12403

Taylor, S. E. (1983). Adjustment to threatening events: A theory of cognitive adaptation. American Psychologist, 38(11), 1161-1173. doi: 10.1037/0003-066X.38.11.1161

Timmerman, M. E., \& Lorenzo-Seva, U. (2011). Dimensionality assessment of ordered polytomous items with parallel analysis. Psychological Methods, 16(2), 209-220. doi: 10.1037/a0023353

Turriff, A., Levy, H. P., \& Biesecker, B. (2015). Factors associated with adaptation to Klinefelter syndrome: The experience of adolescents and adults. Patient Education and Counseling, 98(1), 90-95. doi: 10.1016/j.pec.2014.08.012

Truitt, M., Biesecker, B., Capone, G., Bailey, T., \& Erby, L.
(2012). The role of hope in adaptation to uncertainty: The experience of caregivers of children with Down syndrome. Patient Education and Counseling, 87(2), 233-238. doi: 10.1016/j.pec.2011.08.015

World Health Organization. (2009). Process of translation and adaptation of instruments. Recuperado de http://www.who.int/substance_abuse/research_tools/ translation/en

Yanes, T., Humphreys, L., McInerney-Leo, A., \& Biesecker, B. (2017). Factors associated with parental adaptation to children with an undiagnosed medical condition. Journal of Genetic Counseling, 26(4), 829-840. doi: 10.1007/s10897-016-0060-9

Yopp, J. M., Deal, A. M., Nakamura, Z. M., Park, E. M., Edwards, T., Wilson, D. R. ... \& Rosenstein, D. L. (2019). Psychological and parental functioning of widowed fathers: The first two years. Journal of Family Psychology, 33(5), 565. 
Anexo

\section{Escala de Adaptación Psicológica al Asesoramiento Genético (EAP-AG) \\ Vázquez, Ortega, Scavone, Samaniego, \& Arberas, 2020 \\ (Validación de la Psychological Adaptation Scale, Biesecker et. al, 2013)}

Vivir con

Ser cuidador de un niño con me ha...

\begin{tabular}{|c|c|c|c|c|c|}
\hline & $\begin{array}{c}\text { De } \\
\text { ningún } \\
\text { modo }\end{array}$ & Un poco & Algo & Bastante & Mucho \\
\hline 1. Me ayudó a aceptar el modo en que funcionan las cosas. & 1 & 2 & 3 & 4 & 5 \\
\hline 2. Me ayudó a lidiar mejor con la incertidumbre. & 1 & 2 & 3 & 4 & 5 \\
\hline 3. Me ayudó a adaptarme a lo que no puedo cambiar. & 1 & 2 & 3 & 4 & 5 \\
\hline 4. Me ayudó a aceptar las cosas como son. & 1 & 2 & 3 & 4 & 5 \\
\hline 5. Me ayudó a manejar momentos difíciles. & 1 & 2 & 3 & 4 & 5 \\
\hline 6. Me ayudó a sentirme más cómodo con quien soy. & 1 & 2 & 3 & 4 & 5 \\
\hline 7. Me ayudó a volverme en una persona más fuerte. & 1 & 2 & 3 & 4 & 5 \\
\hline $\begin{array}{l}\text { 8. Me ayudó a sentirme mejor en mi habilidad para } \\
\text { sobrellevar mis problemas. }\end{array}$ & 1 & 2 & 3 & 4 & 5 \\
\hline 9. Me ayudó a volverme una mejor persona. & 1 & 2 & 3 & 4 & 5 \\
\hline 10. Ayudó a que mis relaciones se vuelvan más significativas. & 1 & 2 & 3 & 4 & 5 \\
\hline $\begin{array}{l}\text { 11. Me ayudó a estar más cerca de las personas que me } \\
\text { importan. }\end{array}$ & 1 & 2 & 3 & 4 & 5 \\
\hline $\begin{array}{l}\text { 12. Me ayudó a darme cuenta del amor y el apoyo de otras } \\
\text { personas. }\end{array}$ & 1 & 2 & 3 & 4 & 5 \\
\hline 13. Me ayudó a aprender que mi vida tiene sentido. & 1 & 2 & 3 & 4 & 5 \\
\hline 14. Me brindó un mayor aprecio por la vida. & 1 & 2 & 3 & 4 & 5 \\
\hline $\begin{array}{l}\text { 15. Me ayudó a desarrollar un sentido más profundo del } \\
\text { propósito de la vida. }\end{array}$ & 1 & 2 & 3 & 4 & 5 \\
\hline
\end{tabular}

